\title{
Radiation dose-response curves: cell repair mechanisms vs. ion track overlapping ${ }^{\star}$
}

\author{
Agata Kowalska $^{1}$, Konrad Czerski ${ }^{2}$,, Elena Nasonova ${ }^{3}$, Polina Kutsalo ${ }^{3}$, and Eugen Krasavin ${ }^{3}$ \\ ${ }^{1}$ Division of Physics and Chemistry, Faculty of Marine Engineering, Maritime University of Szczecin, Wały \\ Chrobrego 1-2, 70-500 Szczecin, Poland \\ ${ }^{2}$ Institute of Physics, University of Szczecin, Wielkopolska 15, 70-451 Szczecin, Poland \\ 3 Joint Institute for Nuclear Research, Joliot-Curie 6, 141980 Dubna, Russia
}

Received 5 January 2017 / Received in final form 24 September 2017

Published online 19 December 2017

(C) The Author(s) 2017. This article is published with open access at Springerlink.com

\begin{abstract}
Chromosome aberrations in human lymphocytes exposed to different doses of particle radiation: $150 \mathrm{MeV}$ and spread out Bragg peak proton beams, $22 \mathrm{MeV} / \mathrm{u}$ boron beam and $199 \mathrm{~V} / \mathrm{u}$ carbon beam were studied. For comparison, an experiment with ${ }^{60} \mathrm{Co} \gamma$-rays was also performed. We investigated distributions of aberration frequency and the shape of dose-response curves for the total aberration yield as well as for exchange and non-exchange aberrations, separately. Applying the linear-quadratic model, we could derive a relation between the fitted parameters and the ion track radius which could explain experimentally observed curvature of the dose-response curves. The results compared with physical expectations clearly show that the biological effects of cell repair are much more important than the ion track overlapping.
\end{abstract}

\section{Introduction}

Damage of cells and tissues induced by ionizing radiation has different scenarios and mechanisms which depend on physical properties of investigated beam, delivered dose, dose rate, but also depend on biological response of considered cell type [1-3]. Radiation response is often studied by means of survival curves describing the fraction of cells which sustained given radiation doses. However, a much more reliable indicator of radiation action can be study of chromosome aberrations (CA), which are directly correlated to the processing of the DNA double strand breaks (DSB). Thus, analysis of CA dose-response curves measured in human peripheral blood lymphocytes is one of the most valuable biodosimetric methods. Additionally, the lymphocytes are easily available $\mathrm{G}_{0}$ synchronized human cells and they represent a model of the most radio-sensitive human tissue - bone marrow.

Rapid development of hadron radiotherapy in the last decades increased importance of the radiobiological research and understanding of the physical and biological response to ionizing radiation. On the one hand, physical conditions like formation of ion tracks [4] and the processes of the DNA damage leading to chromosomal aberrations $[5,6]$ are relatively well known. On the other hand, the results of radiobiological experiments still cannot be correctly anticipated. Recent Monte Carlo simulations starting with individual cross section values of traversing

\footnotetext{
* Contribution to the Topical Issue "Dynamics of Systems at the Nanoscale", edited by Andrey Solov'yov and Andrei Korol.

${ }^{a}$ e-mail: konrad.czerski@usz.edu.pl
}

ions and reproducing the track-structure [7] have achieved some remarkable efforts in describing the initial DNA damage, but they do not take into account the biological repair mechanisms which are crucial for understanding final biological outcomes of irradiation - the number of chromosomal aberration or the shape of survival curves. Some comprehensive models - for example the multiscale approach $[8,9]$ implement some additional effects and corresponding parameters describing biological as well as chemical and thermo-mechanical processes like the influence of oxygen enhancement ratio and free radicals propagation via shock waves. Other, more phenomenological models simply compare effects of different radiation quality assuming the ionization density as the most significant parameter describing the biological effects. For example, the local effect model (LEM) which uses the amorphous track structure to predict the shape of cell survival curves [10] and is applied for planning of the hadron therapy tends to overestimate the relative biological effectiveness (RBE) for particles lighter than carbon ions. Recently proposed version of LEM [11] including the DNA giant loops, somehow improves the prediction of experimental data for light ions. Nevertheless, the problem is still not fully solved and some additional adjustable parameters including the repair mechanisms are needed.

Likewise, there are experimental works which extract information from the experimentally obtained doseresponse or survival curves and describe the data by the linear-quadratic model (LQM). For example, the tissue specific linear-quadratic (LQ) parameters determined for photon irradiation enabled to predict the RBE of protons [12]. Fitted parameters of CA dose-response curves and 
statistical distributions of CA [13] have lately allowed to extract some information about physical and biological mechanisms, as well.

The quadratic parameter of the response-curves may have several origins. One of them is the occurrence of efficient DNA repair mechanisms [14] the strength of which depends on the ionization density and local dose distribution [15]. The other, postulated by [16], has a physical origin and can be interpreted as an overlapping of ionization regions of neighboring ion tracks created by incident heavy charged particles. The range of interaction regions is determined by the distribution of fast $\delta$ electrons. Such electrons when overlap the original particle track or other secondary electron path are capable to increase the number of DNA breakpoints [16] which - in consequence leads to additional upward curvature of the dose response.

In the present work, we have further developed the idea of a model independent description of dose-effect curves by introducing an effective interaction radius based on some new experimental data. Our purpose was to introduce a simple physical model of overlapping ion tracks to answer the question how large the ion track radius should be to explain the observed LQ response function. The model is based on the assumption of absorption of a double dose in the overlapping track areas. The simplicity of the model allows us to compare the value of the experimentally determined effective radius to the physical one resulting from the dose distribution of free electrons created within the ion track.

\section{Materials and methods}

\subsection{Blood irradiation and cytogenetic analysis}

All blood samples were obtained from informed, healthy volunteers. Ethical approval was obtained for this study and all participants gave informed consent. Blood was collected by venipuncture into heparinized vacuum containers. Irradiation was done at room temperature.

\subsubsection{Proton beam}

Protons were delivered by Phasotron placed at the Dezheleplov Laboratory of Nuclear Problems of the Joint Institute for Nuclear Research, Dubna, Russia. Samples were irradiated at the medical complex, in the proton therapy room. First set of whole blood samples was exposed to unmodified beam of $150 \mathrm{MeV}$ protons (average LET = $0.57 \mathrm{keV} / \mu \mathrm{m}$; dose rate $0.7 \mathrm{~Gy} / \mathrm{min})$.

Second set was placed at the central region of the Bragg peak, spread out to $10 \mathrm{~mm}$ width by ridge filter RF11 [17]. Average LET of SOBP protons was $1.4 \mathrm{keV} / \mu \mathrm{m}$ (corresponding to the mean energy of about $10 \mathrm{MeV}$ ). Dose rate in the target volume amounted to $1.3 \mathrm{~Gy} / \mathrm{min}$.

\subsubsection{Carbon beam}

Whole blood samples were exposed to $199 \mathrm{MeV} / \mathrm{u}^{12} \mathrm{C}$ beam (pulse duration $500 \mathrm{~ns}$ ) extracted from the ITEPTWAC synchrotron (Russia, Moscow) [18]. Irradiation cuvettes were placed in the plateau region of the Bragg curve where LET did not change significantly and amounted to $16 \mathrm{keV} / \mu \mathrm{m}$.

As a reference radiation ${ }^{60} \mathrm{Co} \gamma$-rays obtained on the remote radiation therapy unit ROKUS-M were used (dose rate $0.82 \mathrm{~Gy} / \mathrm{min}, \mathrm{LET}=0.2 \mathrm{keV} / \mu \mathrm{m})$.

Blood exposed to ${ }^{60} \mathrm{Co} \gamma$-rays, ${ }^{12} \mathrm{C}$ ions, $150 \mathrm{MeV}$ and SOBP protons was placed in $0.5 \mathrm{ml}$ plastic Eppendorf tubes. After the exposure, the whole blood samples were diluted in $4.5 \mathrm{ml}$ of nutrient medium (RPMI supplemented by $20 \%$ foetal calf serum, $2 \mathrm{mM}$ L-glutamine, $100 \mathrm{U} / \mathrm{ml}$ penicillin, $100 \mu \mathrm{g} / \mathrm{ml}$ streptomycin and $1.5 \%$ phytohaemagglutinin) and incubated $48 \mathrm{~h}$ at $37^{\circ} \mathrm{C}$ and $5 \% \mathrm{CO}_{2}$.

\subsubsection{Boron beam}

Boron exposure was conducted at the Flerov Laboratory of Nuclear Reactions, JINR (Dubna, Russia), where monoenergetic $22.1 \mathrm{MeV} / \mathrm{u}^{11} \mathrm{~B}$ beam (LET = $76 \mathrm{keV} / \mu \mathrm{m})$ was delivered by the MC-400 cyclotron.

Human peripheral blood lymphocytes were isolated and highly concentrated in the medium. Concentration of lymphocytes was placed at plexiglas chambers of $1.5 \mathrm{~mm}$ depth, covered by $8 \mu \mathrm{m}$ polycarbonate foil and irradiated. All the ions passed the sample and stopped behind it [19].

Immediately after $\mathrm{B}^{11}$ irradiation lymphocytes were seeded with a density of $0.5 \times 10^{6} / \mathrm{ml}$ in the same nutrient medium and incubated $48 \mathrm{~h}$ at $37^{\circ} \mathrm{C}$ and $5 \% \mathrm{CO}_{2}$.

To accumulate metaphases all samples were treated by colcemid $(200 \mathrm{ng} / \mathrm{ml}) 3 \mathrm{~h}$ prior fixation. Prepared slides were stained in 3\% Giemsa. Chromosome and chromatid type aberrations were scored without karyotyping and classified according to criteria given in [20,21]. For each data point 100-300 metaphases were examined.

Among chromosome-type aberrations dicentrics, paired fragments, centric and acentric rings were scored. The chromatid-type aberrations comprised the chromatid-type exchanges and chromatid-type breaks.

\subsection{Dose-response curves and analytical model of effective track radius}

The yield of CA $Y$ visible after exposure to different doses of ionizing radiation $D$ is usually described by the LQM:

$$
Y=\alpha D+\beta D^{2}
$$

The model up to now remains empirical instead of its great fitting properties. Since both effects biological and physical interfere it has been postulated by Chadwick and Leenhouts [22] that linear coefficient $\alpha$ corresponds to the misrejoining of the DNA breaks arising from single particle track, while quadratic part of the curve described by the $\beta$ coefficient represents misrejoining of two or more DSB's produced due to interaction of independent tracks [16]. Such interaction is more probable for high doses and dose rates when electron tracks of neighboring ions are spatially and temporarily close enough to overlap (see Figs. 1 and 2).

To determine the dose response resulting from overlapping tracks we assume for simplicity that the dose density 


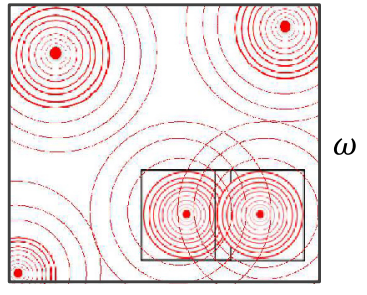

$\omega$

Fig. 1. Schematic view of ion tracks passing through a cell nucleus of a simplified square area $\omega^{2} \sim 25 \mu \mathrm{m}^{2}$ (average area of a human lymphocyte nucleus). Two ion tracks are close enough to overlap.

is constant within an effective track radius. Then, in the case of no interaction between tracks, the aberration yield $Y$ is only proportional to the number of particle tracks $N$, the areal aberration density $A$ and the interaction area $S$ of a single ion track.

$$
Y=A \cdot S \cdot N
$$

Aberration density is linked to the dose density $\rho$ and linear coefficient $\alpha$ of the dose response curve: $A=\alpha \rho$. Thus, the number of aberrations produced by a single track corresponds to $A \cdot S$.

In the case of overlapping ion tracks, we should distinguish between interaction areas of a single ion track $S_{1}$ and overlapping ion tracks $S_{o v}$ with a double dose where of course $S=S_{1}+S_{o v}$. Since the ionization density within the interaction radius of the ion track is constant, the overall aberration yield $Y$ can be written as the sum of aberrations $Y_{1}$ arising from single ion track and from overlapping tracks $Y_{2}$.

$$
\begin{aligned}
Y & =Y_{1}+Y_{2}=\left(N \alpha \rho S-N \alpha \rho S_{o v}\right)+2 N \alpha \rho S_{o v} \\
& =N \alpha \rho S+N \alpha \rho S_{o v} .
\end{aligned}
$$

To determine the overlapping area $S_{o v}$ we ought to know a mean distance between the ion tracks which depends on the irradiation dose applied. As the ion tracks are distributed over the irradiated cell according to the Poisson statistics, the distance between two ion tracks $r$ can be described by the exponential distribution which is analogue to the distribution of the time distance between two events of radioactive decay:

$$
P(r)=\frac{1}{2 \pi L^{2}} e^{-r / L}
$$

where $L$ is a correlation parameter related to the mean value of the ion track distance:

$$
\langle r\rangle=\int_{0}^{\infty} r \frac{1}{2 \pi L^{2}} e^{-r / L} 2 \pi r d r=2 L .
$$

Here, we assumed that $\langle r\rangle$ is much smaller than the cell diameter. The cylindrical symmetry of uniform distributed ion tracks is included by the factor $2 \pi r$. Usually, for the two-dimensional Poisson point process, the a)

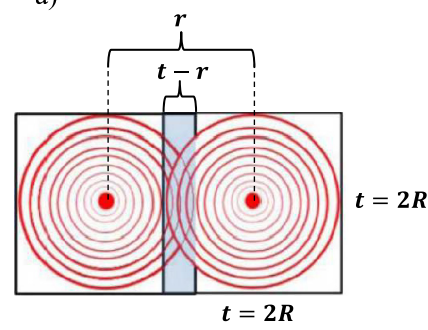

b)

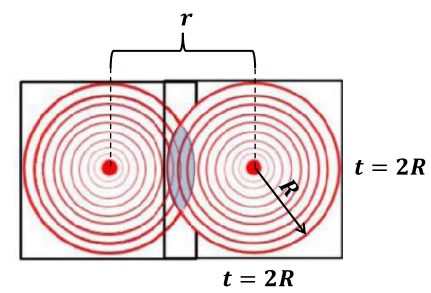

Fig. 2. Schematic view of the overlapping area assessed as a rectangle $S_{o v}^{\prime}$ of $t$ and $t-r$ side length (a) and as a section of two overlapping circular shape tracks $S_{o v}$ (b).

so-called circular void probability [23] giving by the probability density function $2 \pi r e^{-r^{2} / L^{2}}$ is used. In that case, the integrals of overlapping ion track areas cannot be calculated analytically, which is, however, the goal of the present work. The both density functions give within $20 \%$ similar results, which is comparable with other approximations made in the present work.

To determine the correlation parameter $L$, the average area per each ion track which depends on the irradiation dose can be related to the number $N$ of particle traversals per area of cell nucleus $\omega^{2}$ by a simple relation:

$$
\langle r\rangle^{2}=\frac{\omega^{2}}{N}
$$

giving finally

$$
L^{2}=\frac{\omega^{2}}{4 N}
$$

Now, the overlapping area can be calculated as follows:

$$
\begin{aligned}
S_{o v} & =\frac{1}{2 \pi L^{2}} \int_{0}^{2 R} e^{-r / L} s_{o v} 2 \pi r d r \\
& \approx \frac{F}{2 \pi L^{2}} \int_{0}^{t} e^{-r / L}(t-r) t 2 \pi r d r \\
& =F \frac{t^{4}}{6 L^{2}}=F \frac{S^{2}}{6 L^{2}}
\end{aligned}
$$

where the overlapping region $S_{o v}$ is approximated for simplicity by a rectangle (see Fig. 2a) and the interaction radius is given by $R=t / 2$.

The last integral can be easy calculated by expanding the exponential function into the Taylor series (see Appendix A). The result given in the form of an analytical function of $t$ [24] allows to recognize some important dependences and will be very useful for discussion of experimental data. The proportionality factor $F$ should only weakly depend on $L$ (including information on the irradiation dose). It can be determined by a numerical integration assuming the overlapping area to be segments of circles of a radius $R$ :

$$
s_{\text {ov }}=2 R^{2} \arccos \frac{r}{2 R}-r \sqrt{R^{2}-\frac{r^{2}}{4}} .
$$


The results of calculation of the factor $F$ is presented in Figure 3 confirming applicability of approximations performed. We can assume $F=0.58$ in the wide range of $L$.

Coming back to the expression for the aberration yield (Eq. (3)) we obtain

$$
\begin{aligned}
Y & =Y_{1}+Y_{2}=\alpha D+\alpha D F \frac{S}{6 L^{2}} \\
& =\alpha D+\alpha F \frac{4 S}{6 \omega^{2} d} D^{2} .
\end{aligned}
$$

The number of tracks $N$ has been removed due to relation $N=D / d$ where $d$ corresponds to dose deposited by a single ion track within a cell. It corresponds to the ionization density localized along the length $x$ of penetrated lymphocyte nucleus of the material density $\rho_{m}$. It has been assessed that area of a human lymphocyte cell nucleus $\omega^{2}$ is of $25 \mu \mathrm{m}^{2}$ and is almost equal to the cell area [25]. It means that the cell volume can be simplified by a cube of $x \approx 5 \mu \mathrm{m}$ side length (thus $\omega^{2} \approx x^{2} \approx 25 \mu \mathrm{m}^{2}$ ). If we assume a single layer of irradiated cells, the stopping power does not change significantly along the target and the track length is equal to $x$. Assuming that the $N / \omega^{2}$ is the number of ion hits per human lymphocyte nucleus or cell, we can write:

$$
d=\frac{\mathrm{LET} \cdot x}{\rho_{m} \cdot x^{3}}=\frac{\mathrm{LET}}{\rho_{m} \cdot x^{2}} .
$$

Here, we utilized the expression for the linear energy transfer (LET). The formula for the quadratic term of the experimental $\beta / \alpha$ ratio then reads as follows:

$$
\frac{\beta}{\alpha}=\frac{8}{3} \frac{F \rho_{m}}{\mathrm{LET}} R^{2} .
$$

And the final formula describing the effective track radius:

$$
R=\sqrt{\frac{3 \cdot \mathrm{LET}}{8 \cdot F \cdot \rho_{m}}} \cdot \sqrt{\frac{\beta}{\alpha}}
$$

where the relation $S=4 R^{2}$ was used. The expression above can be used for determination of the track radius which could explain the experimentally observed curvature coefficient $\beta / \alpha$. Thus, we will speak about the experimental effective track radius. The physical interaction radius can be calculated by integration of the radial dose distribution (which has a constant dose inside the track core and $\sim 1 / r^{2}$ dependence in the track penumbra) [26] over the whole range of fast $\delta$ electrons. The formula of physical effective radius $R^{\prime}$ (assuming a constant dose density within the ion track) can be then given by:

$$
R^{\prime}=R_{\min } \sqrt{1+2 \ln \frac{R_{\max }}{R_{\min }}}
$$

Radii of the core $R_{\min }$ corresponding to the range of constant dose inside the ion track and penumbra $R_{\max }$,

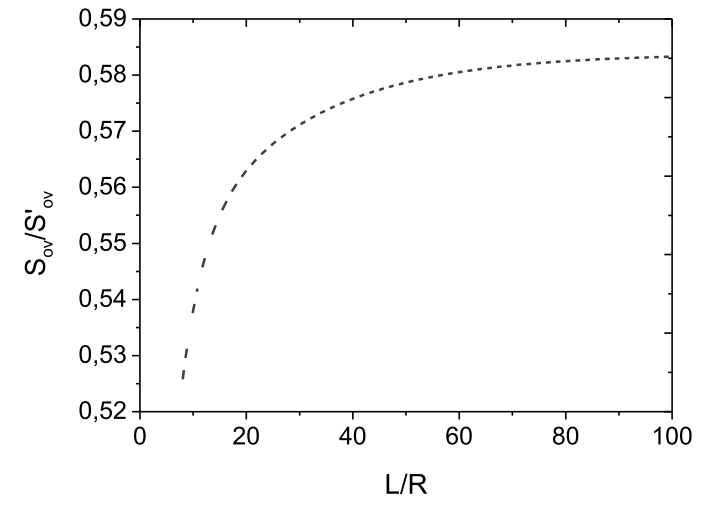

Fig. 3. Proportionality factor $F$ corresponding to the ratio between integrals calculated for circular and rectangle overlapping areas (see Eq. (8)).

corresponding to the range of the $\delta$ electrons are calculated according to the formula proposed by Chatterjee and Schaefer [27] :

$$
R_{\min }=\frac{v}{c} \cdot 0.0116(\mu \mathrm{m})
$$

$$
R_{\max }=0.768 E-1.925 \sqrt{E}+1.257 \mu \mathrm{m} .
$$

Here $E$ stands for particle's kinetic energy per nucleon, $v$ is particle's velocity, $c$ the speed of light. The expressions above are only rough parameterizations and differ from more exact analytical [28] or Monte Carlo calculations [29]. Nevertheless they still deliver useful analytical formulas and enable us to simple estimations. For comparison, we also present $R_{\mathrm{LEM}}$ describing the core radius according to the LEM [10] which reads as follows:

$$
R_{\mathrm{LEM}}=\frac{v}{c} \cdot 0.04(\mu \mathrm{m}) .
$$

$R_{\text {LEM }}$ is assumed to be $40 \mathrm{~nm}$ for $v=c$ and is not motivated physically but gives the best agreement between the model and experimental results.

\section{Results}

Total yield of aberrations per cell depicted in Figure 4 involves chromosome and chromatid aberrations scored without karyotyping. Data obtained for ${ }^{60} \mathrm{Co} \gamma$-rays, $199 \mathrm{MeV} / \mathrm{u}{ }^{12} \mathrm{C}$ ions, $150 \mathrm{MeV}$ and SOBP protons have been fitted by a second order polynomial curve. Total aberration number per cell observed for different doses of $22 \mathrm{MeV} / \mathrm{u}^{11} \mathrm{~B}$ ions represent a linear relation.

We have divided total aberration yield observed after Giemsa staining into exchanges (involving two breaks in one or two chromosomes) and into non-exchange events involving one break in one chromosome. Exchange aberrations comprise dicentrics, acentric and centric rings and chromatid exchanges. Scored non-exchange aberrations were: chromatid breaks and paired fragments. 


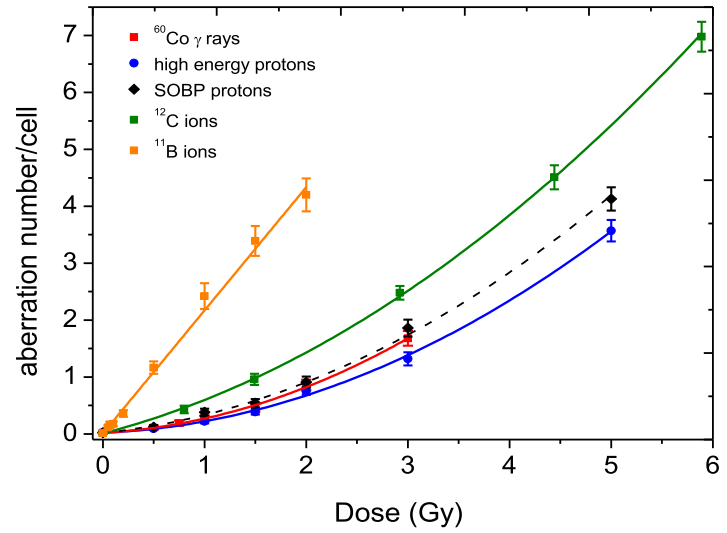

Fig. 4. Dose-effect curve of total aberration yield $Y$ per cell for all considered radiation species. Error bars represent Poisson standard deviation calculated as $\sqrt{Y} / N$, where $N$ represents the number of metaphases scored for each point.

Dose dependence of both aberration types is presented in Figure 5. Parameters of the fits are collected in Table 1.

Comparison of fitting parameters of dose-effect curves obtained for exchange and non-exchange events indicate much more pronounced curvature (represented by $\beta$ parameter) for exchanges. Consequently, linear part of the response curve is mostly affected by chromatid breaks and paired fragments. As it was expected [30], the ratio of exchanges to non-exchange aberration types is smaller for carbon and boron ions than for low LET radiation species. This ratio is constant with the dose in the case of boron (above $\sim 0.3 \mathrm{~Gy}$ ) and carbon ions (above $\sim 0.8 \mathrm{~Gy}$ ) and amounts to 2 . For the dose of $5 \mathrm{~Gy}$ exchanges comprise $73-80 \%$ of total aberration yield for ${ }^{60} \mathrm{Co} \gamma$ rays and both considered proton beams.

The LQM parameters enable to calculate the experimental effective track radius $R$ (Eq. (13)). The corresponding values assuming the circular shape of overlapping tracks (see Fig. 2a) are presented in Table 2. Additionally, track radii calculated according to the LEM [26] denoted as $R_{\mathrm{LEM}}$ and the physical effective radii (Eq. (14)) were determined.

\section{Discussion and conclusion}

In the present work, we determined dose-response curves of human lymphocytes exposed to radiation of different quality $\left({ }^{60} \mathrm{Co}\right.$-gamma radiation, $\mathrm{LET}=0.2 \mathrm{keV} ; 150 \mathrm{MeV}$ protons, $\mathrm{LET}=0.57 \mathrm{keV} / \mu \mathrm{m} ; \mathrm{SOBP}$ protons, LET $=$ $1.4 \mathrm{keV} / \mu \mathrm{m} ;{ }^{12} \mathrm{C}$ ions, LET $=16 \mathrm{keV} / \mu \mathrm{m} ;{ }^{11} \mathrm{~B}$ ions, $\mathrm{LET}=76 \mathrm{keV} / \mu \mathrm{m})$. The LQM was applied to fit the corresponding $\alpha$ and $\beta$ parameters (see Tab. 1). Whereas the linear term ( $\alpha$ parameter) is proportional to the LET value of the particle radiation and corresponds to the number of the DNA DSB induced, the quadratic term ( $\beta$ coefficient) generally results from two different effects: the physical one due to overlapping ion tracks and the biological one. The contribution of track overlapping has been proposed in [16] where an additional portion of positive curvature in the total breakage assessed by mFISH technique was observed. On the other hand, the important role of repair mechanisms has been already shown experimentally [31].

The purpose of the present work was to introduce a simple physical model of overlapping ion tracks to answer the question how large the ion track radius should be to explain the observed LQ response function. The model is based on a concept of the effective track radius and assumption of absorption of a double dose in the overlapping track areas. The radius determined directly from experimental dose-response curves was called the experimental effective track radius. The simplicity of the model allows us to compare the value of the effective radius to the physical one resulting from the dose distribution of fast $\delta$ electrons created within an ion track. The difference between them gives us an estimation of the strength of the biological effects, i.e. mainly the repair mechanisms of the DSB. However, these effects were not discussed in the present paper in detail.

As presented in Table 1 and already in other studies observed [32], the linear term of the LQM is proportional to the LET values of radiations applied. Interesting exceptions are fast protons for which the LET value is more than twice so high as for gamma rays but the $\alpha$ parameter is smaller. In the past, this finding led to speculation that the ionization density induced by protons should be smaller than that suggested by the ion-track model and the energy deposition could take place at larger distances due to electron wake waves or diffusion effects $[8,9,13]$.

As mentioned above, the quadratic term of the LQM reveals both physical and biological effects. The curvature coefficient, the $\beta / \alpha$ ratio, decreases with the increasing LET values and is negligible for the ${ }^{11} \mathrm{~B}$ beam. However, the experimental effective track radii determined by means of equation (13) do not follow this relation. In the case of ${ }^{12} \mathrm{C}$, the experimental radius is larger than for protons. The similar dependence can be also observed for the physical effective radius calculated according to equation (14) and values obtained within the LEM. The physical radii are, however, one order of magnitude smaller than experimentally determined ones. It means that the biological effects clearly dominate. Furthermore, the biological effects should be dose dependent, otherwise we are not able to explain the curvature of the dose-response curves. On the other hand, the fitted $\beta$ values seem to be almost constant, independent of the radiation LET or the physical track radius. The latter additionally supports the biological origin of the curvature of the dose effect curves.

Otherwise, the physical radius $R^{\prime}$ (see Eq. (14)) depends on both the track core radius $R_{\min }$ and the penumbra radius $R_{\max }$ which depend on the velocity and energy of incident particles (Tab. 2). Importance of the penumbra contributions can be documented by a large difference between the $R_{\min }$ and $R^{\prime}$ values. Surprisingly, the ratio between the resulting physical radius $R^{\prime}$ and $R_{\text {min }}$ is almost constant and amounts to about 4 for all irradiations applied. This is clearly result of a very weak dependence of the $R^{\prime}$ value on the penumbra radius $R_{\max }$. A similar factor 4 can be found out when comparing the core radii $R_{\min }$ and $R_{\mathrm{LEM}}$. The increased LEM radius 

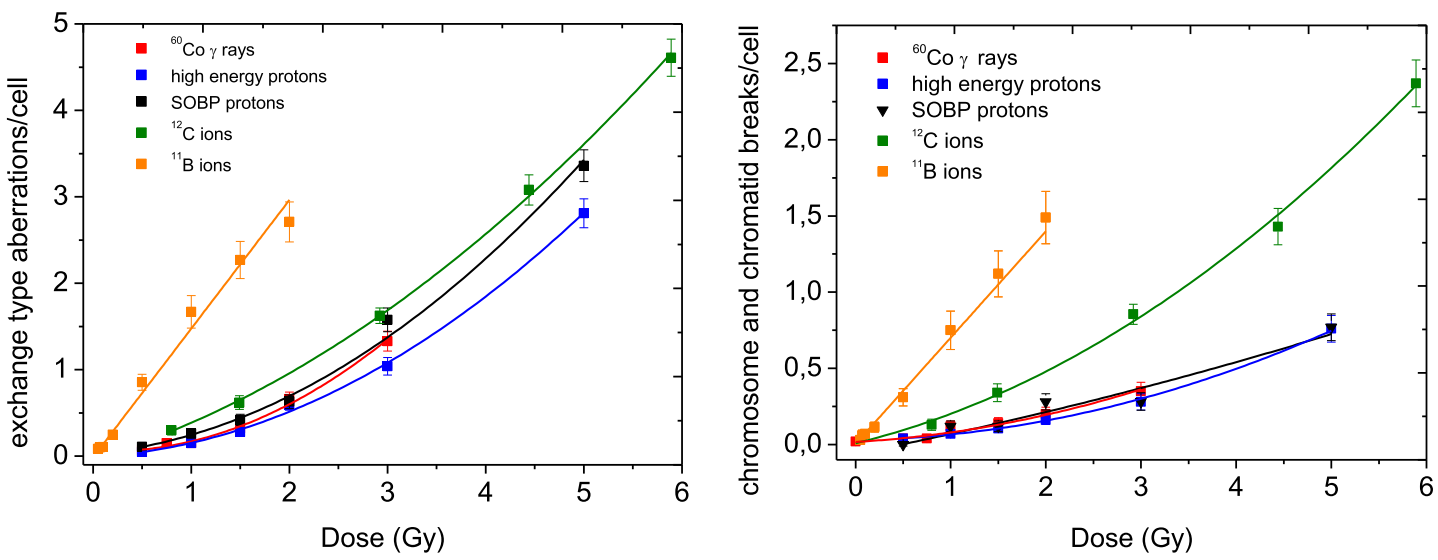

Fig. 5. Left: Total number of exchange type aberrations per cell scored for all considered doses and radiation species. Right: Total number of chromosome and chromatid breaks per cells scored for all considered doses and radiation species. Error bars are calculated according to Poisson statistics.

Table 1. Parameters of fit: linear-quadratic $\left(Y=\right.$ int $\left.+\alpha D+\beta D^{2}\right)$ for ${ }^{60} \mathrm{Co} \gamma$ rays, ${ }^{12} \mathrm{C}$ ions, SOBP and high energy protons; linear $(Y=$ int $+\alpha D)$ for ${ }^{11} \mathrm{~B}$ ions.

\begin{tabular}{lllllll}
\hline Dose-effect curve & Fit parameters & ${ }^{60} \mathrm{Co} \gamma$-rays & High energy protons & SOBP protons & ${ }^{12} \mathrm{C}$ ions & ${ }^{11} \mathrm{~B}$ ions \\
\hline $\begin{array}{l}\text { Chromosome } \\
\text { and chromatid }\end{array}$ & $\alpha$ & $0.036 \pm 0.033$ & $0.01 \pm 0.03$ & $0.12 \pm 0.04$ & $0.15 \pm 0.03$ & $0.69 \pm 0.05$ \\
breaks/cell & int. & $0.026 \pm 0.013$ & $0.027 \pm 0.008$ & $0.0067 \pm 0.0091$ & $0.04 \pm 0.01$ & - \\
& & $0.017 \pm 0.013$ & $0.029 \pm 0.025$ & $-0.06 \pm 0.02$ & $0.009 \pm 0.010$ & $0.004 \pm 0.008$ \\
& $\alpha$ & $-0.05 \pm 0.12$ & $0.058 \pm 0.051$ & $0.10 \pm 0.08$ & $0.34 \pm 0.11$ & $1.48 \pm 0.08$ \\
Exchanges/cell & $\beta$ & $0.16 \pm 0.04$ & $0.10 \pm 0.01$ & $0.12 \pm 0.02$ & $0.08 \pm 0.02$ & - \\
& int. & $0.06 \pm 0.07$ & $-0.006 \pm 0.003$ & $0.03 \pm 0.06$ & $-0.03 \pm 0.11$ & $-0.01 \pm 0.02$ \\
& & & & & & \\
Total aberration & $\alpha$ & $0.096 \pm 0.051$ & $0.07 \pm 0.03$ & $0.18 \pm 0.04$ & $0.46 \pm 0.06$ & $2.17 \pm 0.09$ \\
yield/cell & $\beta$ & $0.15 \pm 0.02$ & $0.13 \pm 0.01$ & $0.13 \pm 0.01$ & $0.12 \pm 0.01$ & - \\
& int. & $0.021 \pm 0.014$ & $0.02 \pm 0.01$ & $0.018 \pm 0.013$ & $0.01 \pm 0.01$ & $0.006 \pm 0.009$ \\
\hline
\end{tabular}

Table 2. Comparison of different effective radii: values of the ion track core $R_{\min }$ and the penumbra $R_{\max }$, physical effective radius $R^{\prime}, R_{\mathrm{LEM}}$ and $\beta / \alpha$ ratios.

\begin{tabular}{lllllll}
\hline Irradiation & $R_{\min }(\mathrm{nm})$ & $R_{\max }(\mu \mathrm{m})$ & $R(\mathrm{~nm})$ & $R^{\prime}(\mathrm{nm})$ & $R_{\text {LEM }}(\mathrm{nm})$ & $\beta / \alpha\left(\mathrm{Gy}^{-1}\right)$ \\
\hline${ }^{60} \mathrm{Co} \gamma$-rays & - & - & $175 \pm 30$ & - & - & $1.6 \pm 0.5$ \\
$150 \mathrm{MeV}$ protons & 5.8 & 93 & $318 \pm 25$ & 23.02 & 20.3 & $1.9 \pm 0.4$ \\
SOBP protons & 2.32 & 8.14 & $287 \pm 28$ & 9.65 & 9.88 & $0.72 \pm 0.13$ \\
${ }^{12} \mathrm{C}$ ions & 6.56 & 126.9 & $620 \pm 50$ & 29.86 & 22.63 & $0.26 \pm 0.04$ \\
${ }^{11} \mathrm{~B}$ ions & 2.55 & 9.65 & - & 10.95 & 8.8 & - \\
\hline
\end{tabular}

probably arises from the fact that larger track radii are necessary to explain experimental $\beta / \alpha$ ratios.

The LQM fits have been separately performed for exchange type of CA and simple breaks, as well. The quadratic parameter $\beta$ obtained for all aberrations is roughly equal to those observed for the exchange type aberrations. Analogously, the linear part of the dose-effect curves is mostly determined by the linear parameter of the non-exchange curves for which repair mechanism are more effective and should be independent of the applied dose. Large contribution of the exchange type aberrations to the total curvature of the dose effect curves underlines once again importance of the biological effects.
To distinguish between the biological and physical components of the dose-effect curves the knowledge about efficiency of repair mechanisms and its dose dependence should be surely determined in separated experiments which are, however, not easy, especially at low doses. The effect of the overlapping ion tracks might be observed in experiments which are able to detect the initial DNA damage, for instance in the premature chromosome condensation (PCC) [33] performed immediately after irradiation. As shown here, there are still open questions with regard of those processes at the microscopic scale and of their interplay with physical and chemical effects, which is of crucial importance for predictive power of the models. 


\section{Author contribution statement}

A. Kowalska and K. Czerski were mainly responsible for modelling and calculations while E. Nasonova, P. Kutsalo and E. Krasavin were mainly responsible for experiments and performed aberration analysis.

Open Access This is an open access article distributed under the terms of the Creative Commons Attribution License (http://creativecommons.org/licenses/by/4.0), which permits unrestricted use, distribution, and reproduction in any medium, provided the original work is properly cited.

\section{Appendix A}

In the following, derivation of the expression for the area of overlapping ion tracks (Eq. (8)) will be given. According to the effective radius approach, we assume that the ionization density within the ion tracks is constant over their rectangular cross sections (see Fig. 2a). Thus, the dose density in the overlapping regions will be doubled. Applying the density probability function (Eq. (4)), we can calculate an average overlapping area between two tracks of side length $t$ and distance $r$, analytically:

$$
\begin{aligned}
S_{o v}^{\prime}= & \frac{1}{2 \pi L^{2}} \int_{0}^{t} e^{-r / L} t(t-r) 2 \pi r d r \\
= & \frac{t}{L^{2}}\left(t \int_{0}^{t} e^{-r / L} r d r-\int_{0}^{t} e^{-r / L} r^{2} d r\right) \\
= & \frac{t}{L^{2}}\left[\left.t\left(-L^{2} e^{-r / L}-L r e^{-r / L}\right)\right|_{0} ^{t}\right. \\
& \left.-\left.\left(-2 L^{3} e^{-r / L}-2 L^{2} r e^{-r / L}-L r^{2} e^{-r / L}\right)\right|_{0} ^{t}\right] \\
= & \frac{t}{L^{2}}\left[L^{2} t-\left(L^{2} t+L t^{2}\right) e^{-t / L}-2 L^{3}\right. \\
& \left.+\left(2 L^{3}+2 L^{2} t+L t^{2}\right) e^{-t / L}\right] \\
= & t\left[(t-2 L)+(t+2 L) e^{-t / L}\right] .
\end{aligned}
$$

Developing $e^{-t / L}$ in the Taylor series, we get:

$$
\begin{aligned}
S_{o v}^{\prime} & =t\left[(t-2 L)+(t+2 L)\left(1-\frac{t}{L}+\frac{t^{2}}{2 L^{2}}-\frac{t^{3}}{6 L^{3}}\right)\right] \\
& =t\left(\frac{t^{3}}{2 L^{2}}-\frac{t^{4}}{6 L^{3}}-\frac{t^{3}}{3 L^{2}}\right)=t\left(\frac{t^{3}}{6 L^{2}}-\frac{t^{4}}{6 L^{3}}\right) \\
& =\frac{1}{6}\left(\frac{t^{4}}{L^{2}}-\frac{t^{5}}{L^{3}}\right) \approx \frac{t^{4}}{6 L^{2}}
\end{aligned}
$$

The overlapping area for more realistic circular cross sections of ion tracks (with radius $R$ ) schematically presented in Figure 2b is given by the integral:

$$
\begin{aligned}
S_{o v}= & \frac{1}{2 \pi L^{2}} \int_{0}^{2 R} r e^{-r / L} \\
& \times\left(2 R^{2} \arccos \frac{r}{2 R}-r \sqrt{R^{2}-\frac{r^{2}}{4}}\right) 2 \pi r d r
\end{aligned}
$$

and can be calculated only numerically. Proportionality factor $F$ (geometry factor) results from the ratio of these two integrals: $S_{o v} / S^{\prime}$ ov (bearing in mind that $t=2 R$ ). Finally, we get very simple expression for the overlapping area:

$$
S_{o v}=F \frac{t^{4}}{6 L^{2}}
$$

with $F=0.58$ (see Fig. 3).

\section{References}

1. A.S. Wozny, G. Alphonose, P. Battiston-Montagne, S. Simonet, D. Poncet, E. Testa, J.B. Guy, C. Rancoule, N. Magné, M. Beuve, C. Rodriguez-Lafrasse, Front. Oncol. 6, 58 (2016)

2. S. Ritter, M. Durante, Mutat. Res. 701, 38 (2010)

3. K.A. George, M. Hada, L.J. Jackson, T. Elliott, T. Kawata, J.M. Pluth, F.A. Cuccinotta, Radiat. Res. 176, $752(2009)$

4. G. Schiwietz, K. Czerski, M. Roth, F. Staunfenbiel, P.L. Grande, Nucl. Instrum. Methods Phys. Res. B 226, 683 (2004)

5. A. Schipler, G. Iliakis, Nucleic Acids Res. 41, 7589 (2013)

6. M. Rall, D. Kraft, M. Volcic, A. Cucu, E. Nasonova, G. Taucher-Scholz, H. Böning, L. Weismüller, C. Fournier, Front. Oncol. 4, 250 (2015)

7. W. Friedland et al., Sci. Rep. 7, 45161 (2017)

8. E. Surdutovich, A.V. Solov'yov, Eur. Phys. J. D 68, 353 (2014)

9. A. Verkhovtsev, E. Surdutovich, A.V. Solov'yov, Sci. Rep. 6, 27654 (2016)

10. T. Elsässer, M. Krämer, M. Scholz, Int. J Radiat. Oncol. Biol. Phys. 71, 866 (2008)

11. F. Tomassino, T. Friedrich, U. Scholz, G. Taucher-Scholz, M. Durante, M. Scholz, Radiat. Res. 180, 524 (2013)

12. M. Wedenberg, B.K. Lind, B. Hrådemark, Acta Oncol. 52, $580(2013)$

13. A. Kowalska, K. Czerski, M. Kaczmarski, M. Lewocki, B. Masojć, A. Łukowiak, Eur. Phys. J D 69, 79 (2015)

14. A. Schipler, G. Iliakis, Nucl. Acids Res. 41, 7589 (2013)

15. M. Scholz, in Microdosimetric response of physical and biological systems to low- and high-let radiations: theory and applications to dosimetry, edited by Y. Horovitz, 1st edn. (Elsevier, Amsterdam, 2006)

16. B.D. Loucas, M. Durante, S.M. Bailey, M.N. Cornforth, Radiat. Res. 179, 9 (2013)

17. J. Kubancak, A.G. Molokanov, JINR Report (2013), http: //www1.jinr.ru/Preprints/2013/077(P16-2013-77).pdf

18. N.V. Markov, A.V. Bakhmutova, A.A. Golubev, A.V. Kantsyrev, V.E. Luckjashin, I.V. Rudskoi, G.N. Smirnov, 
A.D. Fertman, A.V. Khudomyasov, Instrum. Exp. Tech. 57, 55 (2014)

19. A.A. Bezbakh, V.B. Zager, G. Kamiński, A.I. Krylov, Y.G. Teterev, G.N. Timoshenko, Phys. Part. Nucl. Lett. 10, 175 (2013)

20. IAEA, Cytogenetic dosimetry: application in preparedness for and response to radiation emergencies (IAEA, Vienna, 2011)

21. J.R.K. Savage, J. Med. Genet. 12, 103 (1975)

22. K.H. Chadwick, H.P. Leenhouts, Phys. Med. Biol. 13, 78 (1973)

23. A. Baddeley, in Spatial Geometry, edited by W. Weil, 1st edn. (Springer-Verlag, Berlin, Heidelberg, 2007)

24. A. Kowalska, E. Nasonova, K. Czerski, P. Kutsalo, W. Pereira, E. Krasavin, Radiat. Environ. Biophys., submitted
25. R. Anderson, S. Marsden, E. Wright, M. Kadhim, D. Goodhead, C. Griffin, Int. J. Radiat. Biol. 76, 31 (2000)

26. M. Scholz, G. Kraft, Adv. Space Res. 18, 5 (1996)

27. A. Chatterjee, H.J. Schaefer, Radiat. Environ. Biophys. 13, 215 (1976)

28. M.P.R. Waligórski, R.N. Hamm, R. Katz, Nucl. Tracks Radiat. Meas. 11, 309 (1986)

29. F.A. Cucinotta, H. Nikjoo, D.A. Goodhead, Radiat. Environ. Biophys. 38, 81 (1999)

30. S. Ritter, E. Nasonova, M. Scholz, W. Kraft-Weyrather, G. Kraft, Int. J. Radiat. Biol. 69, 155 (1996)

31. G. Iliakis, R. Okayasu, Int. J. Radiat. Biol. 53, 729 (1990)

32. T. Friedrich, U. Scholz, T. Elsässer, M. Durante, M. Scholz, J. Radiat. Res. 54, 494 (2013)

33. J. Deperas-Standył, R. Lee, E. Nasonova, S. Ritter, E. Gudowska-Nowak, Adv. Space Res. 50, 584 (2012) 